\title{
Ballistic interval
}

\author{
Pierre-Marie Gagey
}

\section{ABSTRACT}

We lack a stabilometric parameter that gives us information about the reaction speed of the upright postural control system. This article provides a way to extract this information from the diffusion analysis where it is present. No clinical application of this new parameter can be provided. It is only observed that it is redundant with no conventional parameter.

Keywords: Signal analysis; Reaction speed; stabilometry

\section{INTRODUCTION}

We lack a stabilometric parameter that gives us information on the reaction time of the upright postural control system, and yet this information is contained in an analysis of this signal, known and used since 1993, the 'diffusion analysis' of the time series of the positions of the center of pressure. ${ }^{(1)}$ The question is simply to find the best way to extract this information from this analysis.

\section{The information contained in the diffusion analysis}

The diffusion analysis shows that the stabilometric signal comprises two parts (figure 1), which include first the system states separated by time intervals of less than one second, the observed phenomena there are random ${ }^{(1)}$ whereas the phenomena observed in the other part, beyond a second, are controlled. The interpretation of this analysis by Collins and Coll. is confirmed by the statistical analysis of the amplitude spectrum of the signal(2) ${ }^{(2)}$ hich already has showed a break, around 1 hertz, between stability and stabilization. ${ }^{(3)}$ Moreover diffusion analyses made on the time series of the positions of the center of gravity contain only one part (Gagey, unpublished).

One can notice that all the experimental points of the diffusion analysis represent a covered distance based on a time, so the equation with dimensions is that of a speed, $\mathrm{LT}^{-1}$, a stabilization speed for the points of the first part.

Collins et al. ${ }^{(4)}$, then Lacour et al. ${ }^{(5)}$ found that the experimental curve of the diffusion analysis varied fairly systematically with the age of the subjects (Figure 2).

In Figure 2, it is observed that the same speeds (pointed on the diagram by a small arrow) on the curve of the older subject and on the curve of the younger subject are reached more quickly by the older subject. So what differentiates these subjects is the acceleration of the stabilization phenomena.

\section{Extracting this information}

Extracting this information from the experimental curve of Collins would undoubtedly have been complicated if Ouaknine had not helped us, noticing that Collins' curve is topologically equivalent to the autocorrelation function (figure 3 ) and that the equations of both curves are very similar. ${ }^{(6)}$

On the autocorrelation curve of the acceleration of the center of gravity, it is then possible to look for this difference highlighted by Collins et al., between younger and older subjects. To measure this difference, Collins used the slope of the least square line of the first part of the diffusion curve. With the autocorrelation curve, it is usual and much simpler to use the time shift corresponding to a given value of the correlation coefficient.

This discriminating value of the correlation coefficient was experimentally determined from a cohort of 35 elderly subjects ( $85 \pm 8$ years) $)^{(7)}$ and from a cohort of 116 younger ones ( $26 \pm 7$ years). ${ }^{(8)}$ After checking by a principal components analysis that the acceleration of the center of gravity made a clear distinction between both cohorts (figure 4), a comparison of the autocorrelation curves of the acceleration of the center of gravity of these cohorts showed an area where they were fairly well distinct (figure 5) at the intersection of these curves with the 0.5 value of the correlation coefficient.

We therefore propose to define a marker of the reaction time of the subject (figure 6) by the time delay, $\Delta t$, corresponding to the 0.5 crossing of the autocorrelation curve of the acceleration of the subject's center of gravity. 


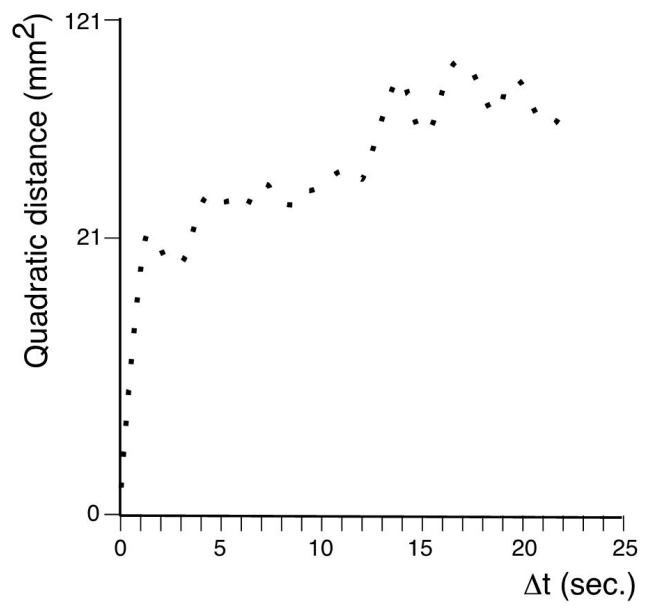

Figure 1. Diffusion analysis of the pressure center vector of a normal subject. The first part of the signal corresponding to time differences, $\Delta t$, less than one second, shows stochastic events, according to Collins and De Luca ${ }^{(1)}$, confirmed by Gagey et al. ${ }^{(2)}$

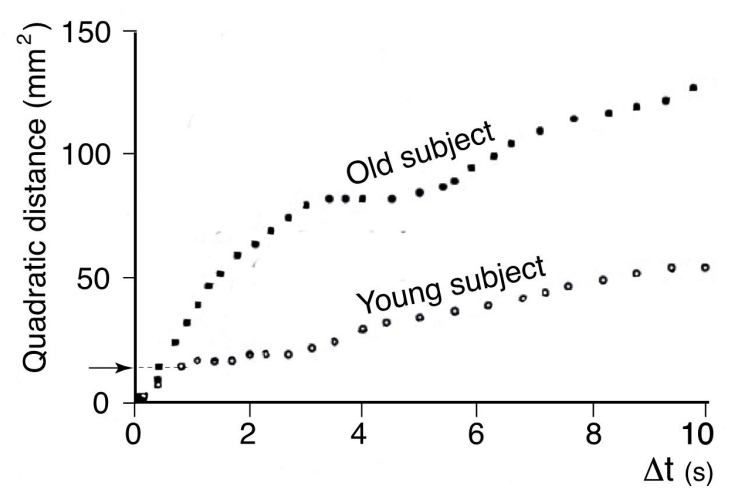

Figure 2. Comparison of the diffusion analysis of an old subject and of a young subject (according to Collins et al., ${ }^{(4)}$ ) The small arrow on the distance scale, points a similar position on both curves but reached more quickly by the older subject. This same distance is reached in both cases for a time interval of less than one second.

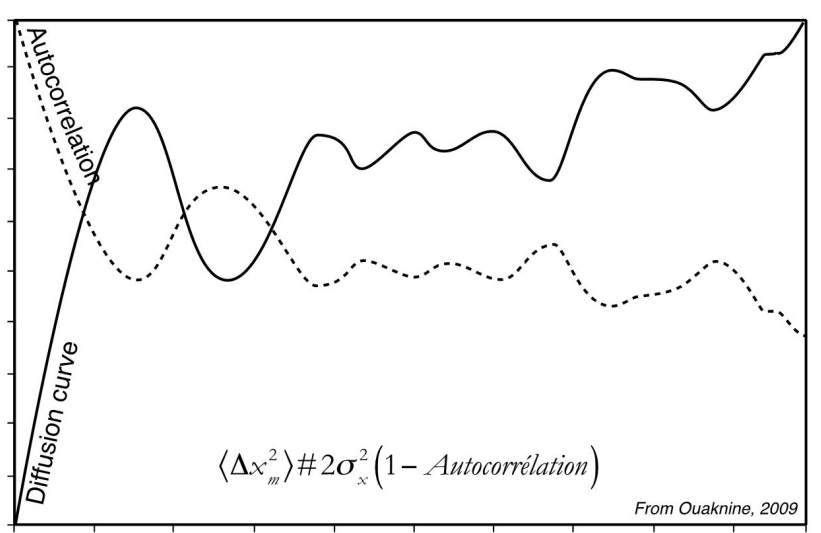

Figure 3. Diffusion curve and autocorrelation. The diffusion curve and that of the autocorrelation are topologically equivalent and their equations are almost identical(6), (this scheme was redone from the post $\left.2009^{(6)}\right)$.

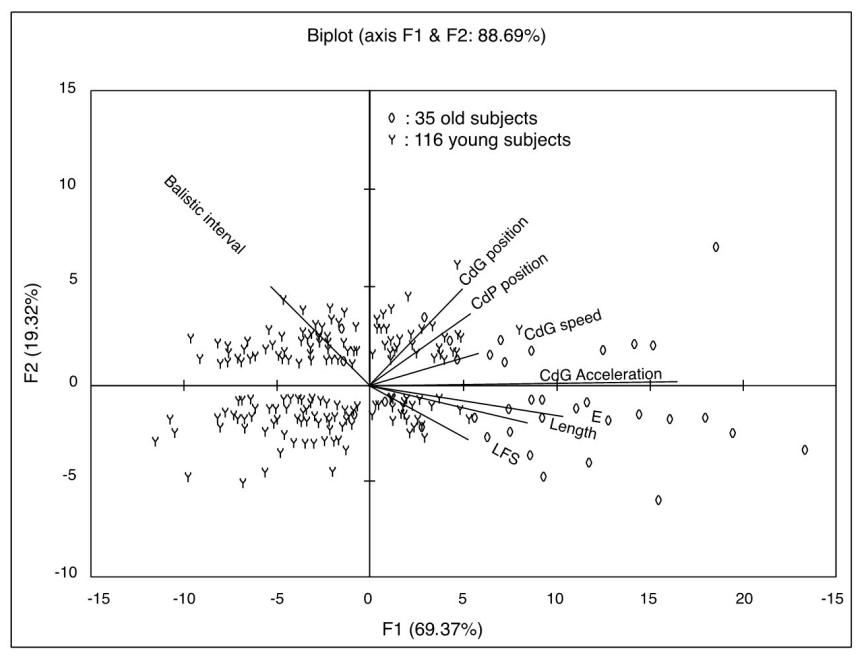

Figure 4. Principal components analysis of the age factor in two modalities: young $(\mathrm{y})$ and old (o). The names of the eight independent variables are written to the extension of their axis. Both cohorts are clearly distinguished along the axis 1 , highly correlated to the acceleration of the center of gravity $(0.965)$.

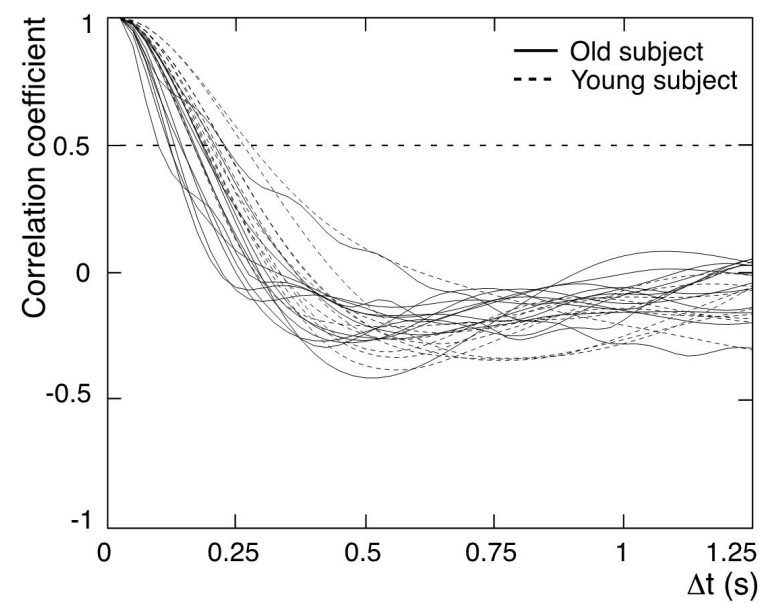

Figure 5. Comparison of the autocorrelation curves of the acceleration of the center of gravity of young subjects and elderly ones. The comparison is limited to 1.25 seconds of time shift.

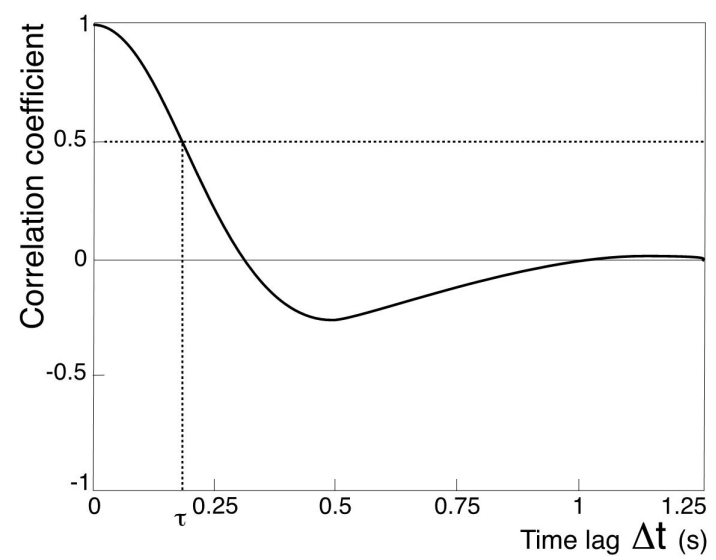

Figure 6. Definition of the parameter. The parameter value is that of the abscissa, $\tau$, of 0.5 crossing of the autocorrelation curve of acceleration of the subject's center of gravity. 


\section{DISCUSSION}

The proposed marker of the postural reaction time is expressed through a time function of the acceleration of the center of gravity, which encourages us to consider how this acceleration varies with time (figure 7). We see a succession of acceleration flashes separated by periods of rest. This phenomenon was described by P. Morasso ${ }^{(9)}$ under mere inspection of the temporal evolution of the position of the center of gravity: some time it stagnates in a very limited area of the support basis, sometimes it is projected to another more or less remote area where it stagnates again for some time. And if we examine the evolution of the acceleration versus time in different subjects, younger, older, elite athletes (figure 7), a clear difference in the duration of the intervals between the bursts of acceleration, according to the subjects, is found; which we propose to name "ballistic intervals."

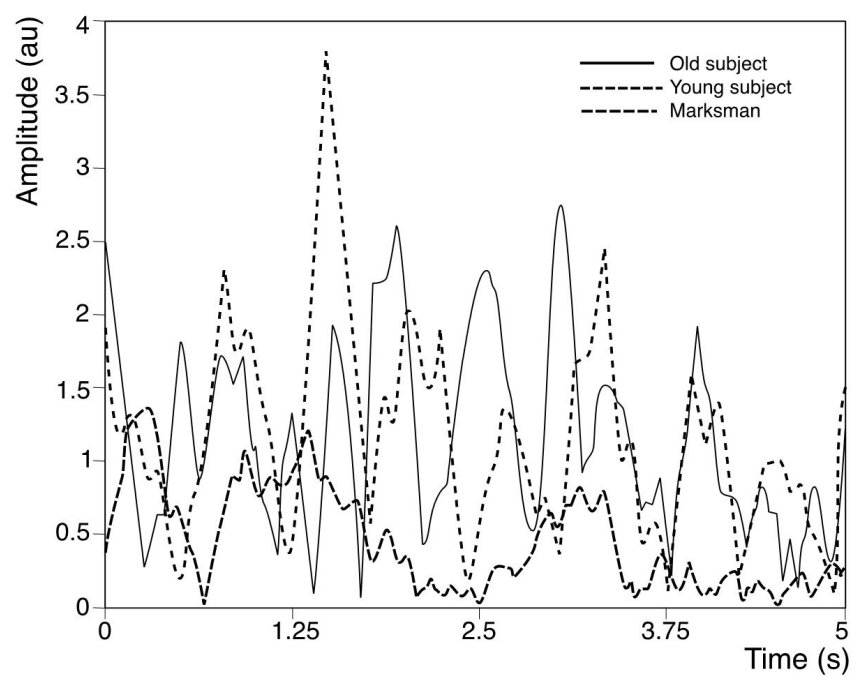

Figure 7. Curves of the acceleration of the center of gravity over time of three different subjects: a top athlete, a young subject and an old one.

\section{CONCLUSION}

A new stabilometric parameter is proposed, the "ballistic interval", represented by the time shift, $\tau$, corresponding to the 0.5 crossing of the autocorrelation function of the acceleration of the center of gravity. This parameter expresses the postural stability of the subject in time; it clearly separates the younger and the older subjects. It is not redundant with another conventional stabilometric parameter.

\section{CONFLICTS OF INTEREST}

The author declare no conflict of interest.

\section{REFERENCES}

1. Collins J, Luca Cd. Open-loop and closed-loop control of posture: a random-walk analysis of center-of-pressure trajectories. Exp Brain Res. 1993;95:308-318.

2. Gagey P, Bizzo G, Debruille O, Lacroix D. The one Hertz phenomenon. In: Igarashi M BF, editor. Vestibular and visual control on posture and locomotor equilibrium. Basel: Karger; 1985. p. 89-92.

3. Winter D, Eng P. Human balance and posture control during standing and walking. Gait \& Posture. 1995;3(4):193-214.

4. Collins J, Luca CD, Burrows A, Lipsitz L. Age-related changes in openloop and closed-loop postural control mechanisms. Exp Brain Res. 1995;104(3):480-492.

5. Lacour M, Bernard-Demanzeb L, Dumitrescu M. Posture control, aging, and attention resources: Models and posture-analysis methods. Clinical Neurophysiology. 2008;38:411-421.

6. Ouaknine $M$. The Brownian diffusion revisited in it's application to postural sway. XIX conference of the ISPGR; 2009 22-25 Juin 2009; Bologna: ISPGR.

7. Boutines C, Gagey B, Gagey P. A "time constant" of the upright postural control system? 2011.

8. Dudde R, Muller G, Bourdeaux O, Weber B, Gagey B, Gagey P. The Marksman's motor control. I) Stabilometric study of the changes during training. 2012 [cited 2014 21/09]; Original work]. Available from: http:// ada-posturologie.fr/The_Marksman.pdf.

9. Baratto L, Morasso P, Re C, Spada G. A new look at posturographic analysis in the clinical context: sway-density vs. other parameterization techniques. Motor control. 2002;6(3):246-270. 